\title{
Tomato Leaf Disease Detection Using Hybrid CNN-RNN Model
}

\author{
Hepzibah Elizabeth David ${ }^{\mathrm{a}, 1}$, K. Ramalakshmi ${ }^{\mathrm{b}}$, R. Venkatesan ${ }^{\mathrm{c}}$, G. Hemalatha ${ }^{\mathrm{d}}$ \\ ${ }^{a, 1, c}$ Department of Computer Science Engineering, Karunya Institute of Technology and \\ Sciences, Coimbatore, India \\ ${ }^{b}$ Department of Computer Science Engineering, Alliance College of Engineering and \\ Design, Alliance University, Bangalore, India \\ ${ }^{d}$ IT Department, University of Technology and Applied Sciences, Oman
}

\begin{abstract}
Tomato crops are infected with various diseases that impair tomato production. The recognition of the tomato leaf disease at an early stage protects the tomato crops from getting affected. In the present generation, the emerging deep learning techniques Convolutional Neural Network (CNNs), Recurrent Neural Network (RNNs), Long-Short Term Memory (LSTMs) has manifested significant progress in image classification, image identification, and Sequence Predictions. Thus by using these computer vision-based deep learning techniques, we developed a new method for automatic leaf disease detection. This proposed model is a robust technique for tomato leaf disease identification that gives accurate and better results than other traditional methods. Early tomato leaf disease detection is made possible by using the hybrid CNN-RNN architecture which utilizes less computational effort. In this paper, the required methods for implementing the disease recognition model with results are briefly explained. This paper also mentions the scope of developing more reliable and effective means of classifying and detecting all plant species.
\end{abstract}

Keywords. Tomato Disease Classification, Hybrid CNN-RNN, CNN, RNN, LSTM

\section{Introduction}

Tomatoes are the most economically essential vegetable crop cultivated worldwide, and the cultivation methods has been improved through the years. Tomato being a commercial and nutritious food crop plays a significant role in agriculture economy.[1] The plant diseases cause economic, agricultural and ecological losses. Plant disease is a major problem which causes serious issues that impairs food security.[15] The tomato crops are infected by diseases due to various factors like climatic conditions, insects, viruses, bacteria. The tomato leaf diseases affect the growth of the plants and the production of tomatoes which results in agricultural and economic loss. Nearly, in India $10-20 \%$ of loss occurs in annual tomato production. Thus, early disease detection prevents the tomato leaf diseases.[5]

\footnotetext{
${ }^{1}$ Hepzibah Elizabeth David, Department of Computer Science Engineering, Karunya Institute of Technology and Sciences, Coimbatore, India; E-mail: hepzibahelizabeth@karunya.edu.in ${ }^{1}$
} 
Image classification has a major role in the identification and detection of the leaf diseases to determine whether the leaf is infected or healthy.[12]

Convolutional Neural Network (CNN) extracts the required image features from an input [6]. Hence it is one of the preferable classification methods in plant leaf disease detection. Major significances of $\mathrm{CNN}$ include natural language processing, medical image analysis, image classification. [3]Recurrent Neural Networks (RNN) learns to recognize image features across time, they extract necessary features from the inputs for further classification. They were designed to deal with problems with sequence prediction.[4] Due to the presence of feedback loops in the recurrent layer, RNN remembers its previous inputs. The LSTM layer in RNN, explicitly known for its memory that helps in maintaining the information for long periods.[7] It has been noticed that LSTMs aid feature extraction capability of CNN used in a layered order as they have the ability to remember a selection of long sequences for long duration of time and CNNs can extract important features out of it.[11]

\section{Related Works}

Plant disease is a major problem in agriculture, that minimizes the quantity of food production and reduces the quality of agricultural products.[13] The heedful disease diagnosis and appropriate handling protects the plants from massive loses. Hence plant disease detection techniques protect the crops from being infected rapidly and improves agricultural growth, the performance of the detection techniques are crucial.[14] Hence the advanced computer vision-based techniques came to compensate the lack of human expertise [4]. In 2011, Neural Network technique has been implemented. This used otsu's method, then the extracted features [5].

In 2012, the Artificial Neural Network (ANN) classifier was used, the images were filtered using the Gabor filter. Then the extracted feature values are trained with the classifier that provides $91 \%$ accuracy. [8]. Thus, in the field of agriculture for plant disease recognition and classification the deep convolutional neural networks are implemented [2]. The deep learning Convolutional Neural Network architecture trained with machine learning based model for predicting the tomato plant disease is proposed [7].

The advancements in CNN eventually lead to the development of deep CNN architectures with various pre-trained models like AlexNet, GoogleNet, DenseNet, ImageNET, VGGNet and ResNet have been used for plant leaf disease. In 2018, the deep Convolutional Neural Network based architecture with VGG for disease diagnosis with $99.58 \%$ of accuracy which is highly potential when trained and provides best performance compared to other models [9]. In 2019, four deep CNN with two models for object detection, the mask R-CNN and faster R-CNN has been implemented for detection of tomato diseases. [10]. A pre-trained CNN with LSTM based model has been implemented for the detection of pests and apple disease which outperforms the deep CNN based architectures [6]. In 2020, a robust model with RNN based architecture for rapid detection of infected regions in the plants has been introduced [4]. 


\section{Proposed System for Tomato Leaf Disease Detection}

The previously implemented traditional leaf disease detection models classified the diseases with better performance. These implementations indicateneed for early detection. However, those existing models failed to detect the disease in earlier stage.

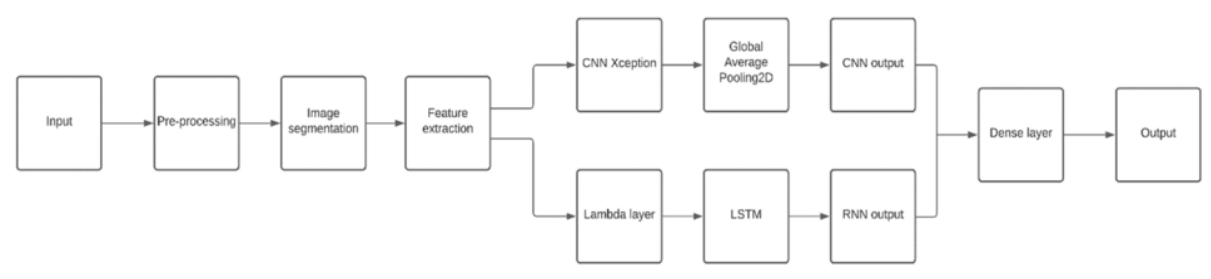

Figure 1. Hybrid CNN-RNN model

Thus, the hybrid CNN-RNN model (Figure 1) has been introduced for the early tomato leaf disease detection. The input image from dataset goes through image pre-processing techniques. Then the image segmentation using label-binarizer is performed. For feature extraction the image pixels (width, height) of each image is taken. The feature values is given to both pre-trained CNN Xception model and RNN model. The outputs from the $\mathrm{CNN}$ and the RNN classifiers is multiplied using element-wise multiplication. Then the output enters into the dense layer. And finally, the required output from the data is predicted using the proposed CNN-RNN model.

\subsection{Implementation Details}

The Plant Village dataset [16] is used for the evaluation of automatic identification and classification of the plant leaf diseases. In this study, 2000 images are taken for training and texting purpose. The folders of tomato leaf disease includes 10 classes of (9 diseases and one healthy class) images are resized to $256 * 256$ pixels as required for the proposed model. The input image from the dataset is pre-processed using the image data generator which flips, rotates and sheers the input image, the values are fixed, such that rotation range $=25$, width_shift range $=0.1$, height shift_range $=0.1$, shear_range $=0.2$. Then the pre-processed image obtained is used further implementation. The proposed model contains both the CNN and RNN deep learning techniques. Both the CNN and the RNN model runs in parallel state. The pre-trained Xception CNN model, which is also an extension of the inception model. It is an efficient architecture that involves in depth wise separable convolutions known for its improved computation time and depends on shortcuts between convolution blocks.. The CNN bottleneck is also a neural network layer present in the inception network, that helps to reduce number of feature maps (channels) in the model, that tends to increase in each layer. It is defined by using the Global Average Pooling 2D layer from the pre-trained model multiplied with CNN's output. After creating the CNN model, the output shape is computed. Parallelly the lambda layers that are useful in saving and inspecting the model in RNN is defined. In Recurrent Neural Network, the rgb to _grayscale inputs, rgb _to_grayscle output shape and the input tensor (width, height, channel_axis) values are acquired. The input shapes are reshaped, with each of the image pixels of $256 * 256$. Two parameters is used for reshaping, that are, number of timesteps and the input dimensions for each timestep. The timesteps value can be of 
$256 * 256=16 * 4096=32 * 2048$. Then the LSTM layer is included in the model. The Xception CNN's output shape is taken as the input. Both the CNN's output and the RNN's output is multiplied with element wise multiplication. The LSTM initiates predictions from the timesteps obtained. Thus, the predictions is made at $\mathrm{x}$ variable for all the classes. The dense layer used, reduces the classes to 10 labels as required and the 'softmax' is used as activation function.

\section{Results}

This section shows the experimental results of the proposed work. This proposed work used $20 \%$ of the leaf image dataset for testing. So, the model was trained using $80 \%$ of the dataset and validated during each epoch. This model was evaluated based on two timesteps 16 and 32, for each timestep four varying epochs were run to fine tune the model. The complete records of the tests carried in the tuning parameters is mentioned in Table.2.

First paragraph.
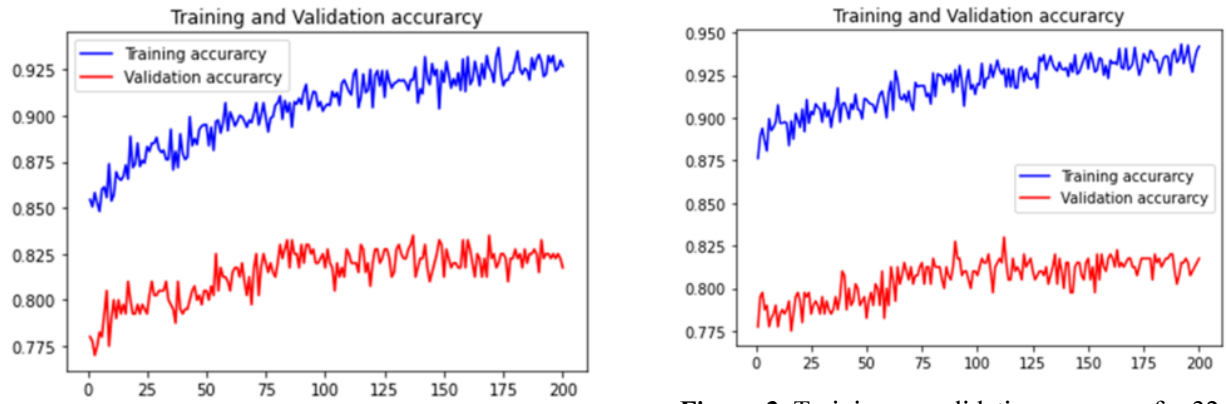

Figure 2. Training vs validation accuracy for 16 timesteps

Figure 3. Training vs validation accuracy for 32 timesteps

Table 2. Experimental Results of the model tested with various parameters for hyper-parameter model tuning.

\begin{tabular}{|c|c|c|c|c|c|c|}
\hline & & Epochs & Accuracy (\%) & $\begin{array}{c}\text { Validation } \\
\text { Accuracy (\%) }\end{array}$ & $\begin{array}{c}\text { Top K } \\
\text { Categorical } \\
\text { Accuracy (\%) } \\
\end{array}$ & $\begin{array}{c}\text { AUC score } \\
(\%)\end{array}$ \\
\hline \multirow{8}{*}{ 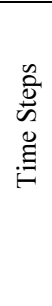 } & \multirow{5}{*}{ 으 } & 50 & 76.75 & 71.95 & 98.25 & 97.19 \\
\hline & & 100 & 78.25 & 74.41 & 97.75 & 96.98 \\
\hline & & 150 & 80.75 & 75.76 & 98.00 & 96.96 \\
\hline & & 200 & 81.75 & 81.44 & 97.50 & 97.17 \\
\hline & & 50 & 76.25 & 71.26 & 97.50 & 96.96 \\
\hline & \multirow[t]{3}{*}{$\pi$} & 100 & 79.00 & 73.79 & 98.00 & 97.35 \\
\hline & & 150 & 80.50 & 76.63 & 98.00 & 97.35 \\
\hline & & 200 & 81.75 & 80.56 & 98.25 & 96.89 \\
\hline
\end{tabular}

From Table. 2, we can observe the model performance and can evaluate the classification capability of the model. The model obtained $98.25 \%$ classification accuracy as the highest among the ten classes it was trained and obtained the best overall accuracy of 81.75 for classification. During training, the model started show to signs of overfitting when it was trained for 300 epochs. The other possibility to increase the performance of the model would be increasing number of timesteps, which can be observed from Figure. 2 and Figure. 3. The model can be again tested with 
possible different timesteps and input dimensions of the model, and we can further conclude may be increase in timesteps would increase the classification accuracy.

\section{Conclusion}

In agriculture, leaf disease detection is crucial and accuracy with good precision for real-time disease detection is required. Hence, it is essential that the model should identify tomato leaf disease at an early stage, which requires minimum time for detection and less computation effort is also needed when compared with other existing traditional methodologies. This paper proposed a hybrid deep learning CNN-RNN classifier for detection of tomato leaf disease and the implementation and performance analysis of the model was also discussed. Due to its high prediction rate, with timeseries data, earlier detection is made possible. The proposed model obtained $98.25 \%$ as the best categorical accuracy and accuracy of $81.75 \%$ for disease classification. In future, this work can be further hold forth by improving the performance and the robustness of the model for real-time application.

\section{References}

[1] Batool, Ayesha, et al. Classification and Identification of Tomato Leaf Disease Using Deep Neural Network.2020 International Conference on Engineering and Emerging Technologies (ICEET). IEEE, 2020 .

[2] Sladojevic, Srdjan, et al. Deep neural networks based recognition of plant diseases by leaf image classification. Computational intelligence and neuroscience 2016 (2016).

[3] Sabrol, H., and K. Satish. Tomato plant disease classification in digital images using classification tree. 2016 International Conference on Communication and Signal Processing (ICCSP). IEEE, 2016.

[4] Lee, Sue Han, et al. Attention-based recurrent neural network for plant disease classification.Frontiers in Plant Science 11 (2020).

[5] Al-Hiary, Heba, et al. Fast and accurate detection and classification of plant diseases.International Journal of Computer Applications 17.1 (2011): 31-38.

[6] Turkoglu, Muammer, Davut Hanbay, and Abdulkadir Sengur. Multi-model LSTM-based convolutional neural networks for detection of apple diseases and pests. Journal of Ambient Intelligence and Humanized Computing (2019): 1-11.

[7] Adhikari, Santosh, et al. Tomato plant diseases detection system using image processing.1st KEC Conference on Engineering and Technology, Lalitpur. Vol. 1. 2018.

[8] Kulkarni, Anand H., and Ashwin Patil. Applying image processing technique to detect plant diseases. International Journal of Modern Engineering Research 2.5 (2012): 3661-3664.

[9] Ferentinos, Konstantinos P.Deep learning models for plant disease detection and diagnosis. Computers and Electronics in Agriculture 145 (2018): 311-318.

[10] Wang, Qimei, et al. Identification of tomato disease types and detection of infected areas based on deep convolutional neural networks and object detection techniques. Computational intelligence and neuroscience 2019 (2019).

[11] Nerkar, Bhavana, and Sanjay Talbar. Fusing Convolutional Neural Networks to Improve the Accuracy of Plant Leaf Disease Classification. Current Journal of Applied Science and Technology (2020): 9-19.

[12] Alimboyong, Catherine R., Alexander A. Hernandez, and Ruji P. Medina. Classification of plant seedling images using deep learning.TENCON 2018-2018 IEEE Region 10 Conference. IEEE, 2018.

[13] Kaya, Aydin, et al. Analysis of transfer learning for deep neural network based plant classification models. Computers and electronics in agriculture 158 (2019): 20-29.

[14] Mkonyi, Lilian, et al. Early identification of Tuta absoluta in tomato plants using deep learning. Scientific African 10 (2020): e00590.

[15] Fuentes, Alvaro, et al. A robust deep-learning-based detector for real-time tomato plant diseases and pests recognition. Sensors 17.9 (2017): 2022.

[16] https:/www.kaggle.com/emmarex/plantdisease 